\title{
THE RATCHETING OF COMPRESSED THERMALLY GROWN THIN FILMS ON DUCTILE SUBSTRATES
}

\author{
M. Y. HE ${ }^{1}$, A. G. EVANS ${ }^{2 \dagger}$ and J. W. HUTCHINSON ${ }^{3}$ \\ ${ }^{1}$ University of California, Santa Barbara, Santa Barbara, CA 93106, USA, ${ }^{2}$ Materials Institute, \\ Princeton University, Princeton, NJ 08540, USA and ${ }^{3}$ Division of Engineering and Applied Sciences, \\ Harvard University, Cambridge, MA 02138, USA
}

(Received 13 December 1999; accepted 8 February 2000)

\begin{abstract}
An analysis of the displacements experienced by undulating thermally grown thin films upon thermal cycling has been presented. The film has been assigned a thermal expansion coefficient that causes it to be compressed upon cooling. It has also been allowed to thicken at high temperature by oxidation of the substrate. It is shown that, in some circumstances, ratcheting occurs, wherein the undulation amplitude, $a$, increases with each thermal cycle. When such a response happens, undesirable cyclic failure modes are induced. The analysis reveals that there is a critical undulation amplitude, $a_{\mathrm{c}}$, below which ratcheting does not occur. This critical size is related to the expansion misfit, the substrate yield strength and the growth strain in the film per cycle. Connections between these variables and $a_{\mathrm{c}}$ are derived. (C) 2000 Acta Metallurgica Inc. Published by Elsevier Science Ltd. All rights reserved.
\end{abstract}

Keywords: Physical vapor deposition; Stress/strain theory; Layered materials; Fatigue

\section{INTRODUCTION}

Thin films on thick, ductile (metal or polymer) substrates when residually compressed by a misfit stress, $\sigma_{0}$, are susceptible to various out-of-plane displacement instabilities [1-9]. The most widely documented examples involve the thin thermally grown oxide (TGO) of $\alpha-\mathrm{Al}_{2} \mathrm{O}_{3}$ that forms on superalloy components used for propulsion and power generation [1-9]. In this example, a top thermal barrier layer may also be present [Fig. 1(a)]. Related phenomena occur around metal interconnects in semiconductor devices [10]. The displacements occur by distorting the substrate. They reduce in-plane compression, but lead to tensile stresses [8] that, in some cases, initiate the failure sequence.

The starting hypothesis is that pre-existing undulations in the film initiate the instability. The undulations increase in amplitude, with time or cycles [Fig. 1(a)], in accordance with two known manifestations. One occurs isothermally, at elevated temperatures, with the displacements accommodated by substrate creep. This phenomenon is often referred to as "wrinkling" [6]. The other occurs upon thermal cycling [11]. Typically, cycling involves a hold time $\Delta t$ at the highest temperature with intervening

$\dagger$ To whom all correspondence should be addressed. up and down ramps. The film thickens during $\Delta t$, because of the kinetics of oxidation. In this manifestation, the undulations are unaffected by time-attemperature. Instead, they accumulate systematically with each cycle. It will be shown that the displacements are enabled by cyclic yielding of the substrate and require that there be an increment in film thickness in each cycle, governed by its thickening kinetics [4-6]. By analogy with related thermomechanical phenomena [12-15], this process is referred to as "ratcheting". This is the mechanism analyzed in the present article.

When ratcheting happens, and a top layer is present, the increase in undulation amplitude upon cycling induces out-of-plane tensile strains, $\varepsilon_{z z}$, in this layer [Fig. 1(a)]. These strains may cause cracks parallel to the interface which, in turn, induce failures, such as those reported for oxide thermal barriers [16]. An example is shown on Fig. 1(b). Moreover, the state of stress in the film changes and becomes tensile at some locations [7, 17]. At these sites, film cracking may occur, despite the overall state of compression.

In general, ratcheting requires the presence of at least two materials having significant differences in thermal expansion coefficient [12, 13]. Then, upon thermal cycling, and in the presence of a strain that provides a directional bias, material displacements occur with each cycle. For any simple thermo-mech- 


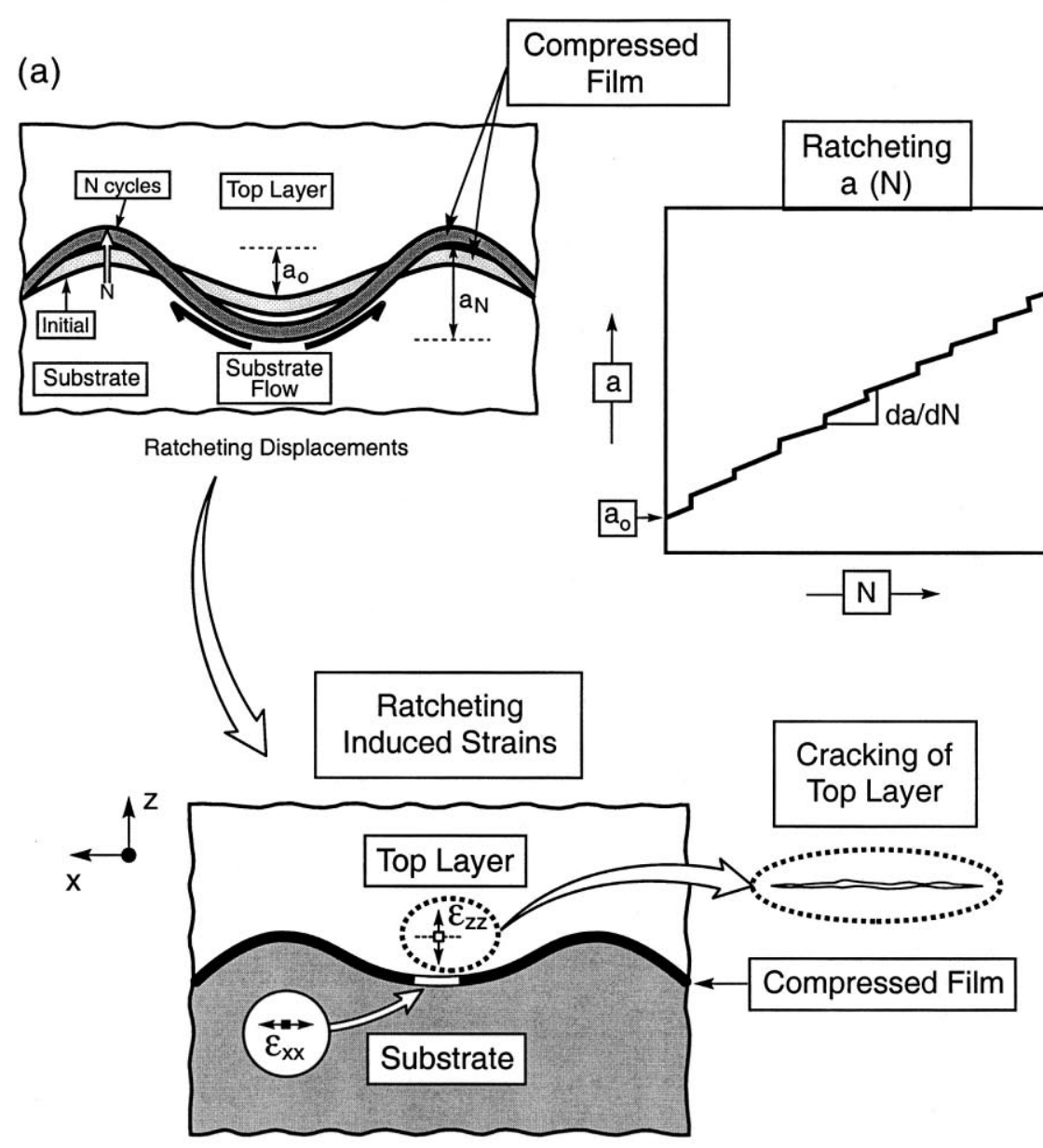

(b)

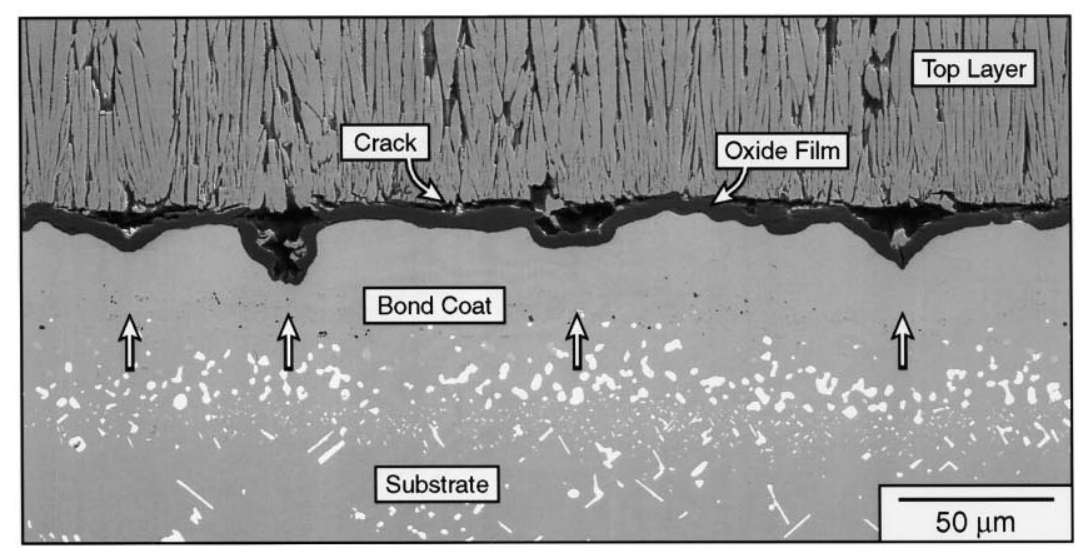

Fig. 1. (a) A schematic indicating the ratcheting phenomenon in thin compressed films and the strain induced in the top layer, which may result in cracking and failure. (b) The undulations in the oxide film, indicated by arrows, grew by ratcheting (they did not exist when the film was deposited). The growth of the undulations caused cracks to initiate in the top layer, in the manner suggested by the schematic (courtesy Dr D. Mumm) 
anical loading, the range of responses has been characterized by a Bree diagram (Fig. 2) [14, 15], wherein the regions that experience elastic response, shakedown and ratcheting are identified. The existence of these regions will re-emerge in the present study, albeit with different co-ordinates. A good example is the ratcheting of particle reinforced metals comprising, say, aluminum alloys with $\mathrm{SiC}$ reinforcements [12]. These ratchet upon thermal cycling when a small stress is applied.

When the film is perfectly planar, the absence of a shear stress in the substrate (except near free edges) means that there can be no out-of-plane response to thermal cycling [1]. However, shear stresses induced in the substrate by film undulations may exceed its yield strength [1]. When this happens, the undulation amplitude enlarges, through substrate plasticity. The extent to which this continues as the system thermally cycles is examined in this study. It will be shown that the ratcheting is transient when the film thickness remains invariant. However, when a thickening occurs in each thermal cycle (because of a growth mechanism such as oxidation), the ratcheting can attain steady state, wherein the amplitude consistently increases with continued cycling. This is the phenomenon of present concern because of its direct association with failure [Fig. 1(a)].

\section{CONCEPTS}

While ratcheting is a documented phenomenon [12-15], the conditions that allow it to occur in thin films on thick substrates have not been explicitly identified. Absent isothermal wrinkling at high temperature [11], cyclic plasticity in the substrate affords the only mechanism capable of providing cumulative displacements. Such responses become

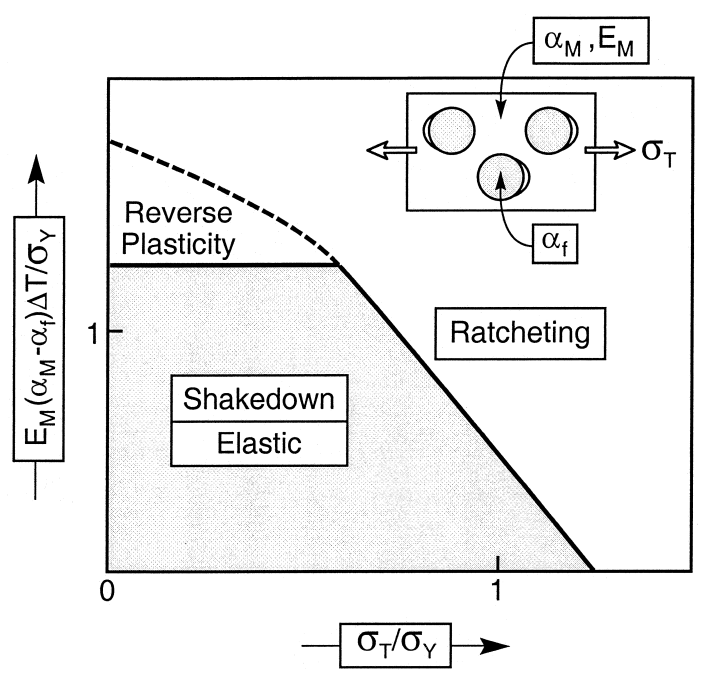

Fig. 2. A Bree diagram developed for transverse loading of a metal matrix composite [12]. possible wherever the equivalent stress, $\sigma_{\mathrm{e}}$, induced in the substrate exceeds its yield strength, $\sigma_{\mathrm{Y}}$. This stress is governed by [1, 3]: the amplitude of the undulations, $a_{0}$, relative to their wavelength, $2 L$, the film thickness, $h$, the relative elastic moduli of film, $E_{\mathrm{f}}$, and substrate, $E_{\mathrm{s}}$, and the misfit stress, $\sigma_{0}$, defined as

$$
\sigma_{0}=\Delta \alpha \Delta T E_{\mathrm{f}} /\left(1-v_{\mathrm{f}}\right)
$$

where $\Delta \alpha$ is the difference in thermal expansion coefficient between the substrate, $\alpha_{\mathrm{s}}$, and the film, $\alpha_{\mathrm{f}}, \Delta T$ is the amplitude of the temperature change (with the highest temperature in the cycle as the reference) and $v_{\mathrm{f}}$ the Poisson ratio for the film. Note that the misfit stress is that in a perfectly flat film subject to $\Delta T$ (not the stress in the substrate).

It is not sufficient to just yield the substrate on initial cooling. Such yielding only produces an initial displacement, followed by either shakedown or cyclic plastic straining of the substrate [3]. That is, ratcheting effects will damp-out after a few cycles. This assertion is verified by some of the calculations that follow. An additional phenomenon is needed for continuous ratcheting. For the particle reinforced metals cited above, this is achieved with an applied stress, which biases the plastic response to the extent needed to induce continuous plastic straining with each thermal cycle [12]. For thin compressed films on thick substrates, the analog is less obvious.

Two high temperature effects operate in thermally grown oxides that might lead to ratcheting. One involves creep in the TGO [18], which would relax the stresses. The other involves the growth strain that occurs upon oxidation [17]. That is, at the highest temperature in the cycle, taken to be $1000^{\circ} \mathrm{C}$, the film thickens during the hold time $\Delta t$ in accordance with oxidation kinetics. The present article demonstrates that ratcheting can arise in situations that involve growth strains (Fig. 3). The philosophy adopted for these calculations is to seek the most basic elements of material behavior that result in ratcheting. Once the principles have been established, explicit determinations of ratcheting rates, with realistic material properties, would follow. The simplest model that can be envisaged comprises a thin film with a smaller thermal expansion coefficient than that for the substrate: with the latter having a temperature independent yield strength, $\sigma_{\mathrm{Y}}$. The elastic moduli of the film, $E_{\mathrm{f}}$, and substrate, $E_{\mathrm{s}}$, are taken to be different and representative of an oxide film on a metal substrate $\left(E_{\mathrm{f}} / E_{\mathrm{s}}=2\right)$.

The calculations are performed by imposing on the film an isotropic growth strain, $\varepsilon_{\mathrm{g}}$, at the highest temperature (Fig. 3), taken to be $1000^{\circ} \mathrm{C}$, then cycling to room temperature and back. This is a stress-free strain, analogous to a thermal expansion. For simplicity, $\varepsilon_{\mathrm{g}}$ is taken to be the same for each cycle. Later embellishments might allow it to 
change as the film thickens. Also, for tractability, the film is considered to be elastic, with a temperature independent modulus $E_{\mathrm{f}}$ (except for the growth strain). The temperature dependence of $E_{\mathrm{f}}$ and creep in the film will be addressed in a subsequent paper.

The major emphasis concerns the dependence of the ratcheting rate on $\varepsilon_{\mathrm{g}}$, as well as the material properties (especially $\sigma_{\mathrm{Y}}$ and $\Delta \alpha$ ) and the film morphology $\left(a_{0} / 2 L\right.$ and $\left.h\right)$. The authors are unaware of an analytical method that could elucidate this role with appropriate accuracy. The approach taken, therefore, is to use numerical methods to find property domains susceptible to ratcheting.

In the following analysis, the top layer depicted on Fig. 1(a) has been neglected, primarily because the material comprising this layer has low shear modulus [19] (created by incorporating inter-columnar porosity to attain strain tolerance). This low modulus would provide minimal resistance to displacements of the film. In subsequent studies, the explicit (though small) influence of this layer will be examined.

\section{RATCHETING ANALYSIS}

\subsection{Method}

The finite element method is employed for the calculations, using the ABAQUS code. The substrate is taken to be 100 times thicker than the film to suppress overall bending of the system. Periodic boundary conditions are used with a unit cell having width, $L$ (Fig. 3). Generalized plane strain conditions account for the bi-axial nature of thermal loading. The growth strain $\varepsilon_{\mathrm{g}}$ is introduced at the temperature maximum $\left(T=1000^{\circ} \mathrm{C}\right)$ in each cycle. This can be accomplished in several ways, depending on the flexibility and options available within the finite element code. A code that incorporates a transformation strain option enables growth to be introduced as a sequence of transformation steps, giving rise to the accompanying elastic and plastic strain increments. When the properties of the film and substrate are temperature-independent, as in the present case, an equivalent approach based on a fictitious temperature change may be used. Then, the growth strain can be achieved by adding an extra temperature change, $\Delta T_{\mathrm{g}}$, at the temperature maximum in each cycle: where $\varepsilon_{\mathrm{g}}=\Delta \alpha \Delta T_{\mathrm{g}}$.

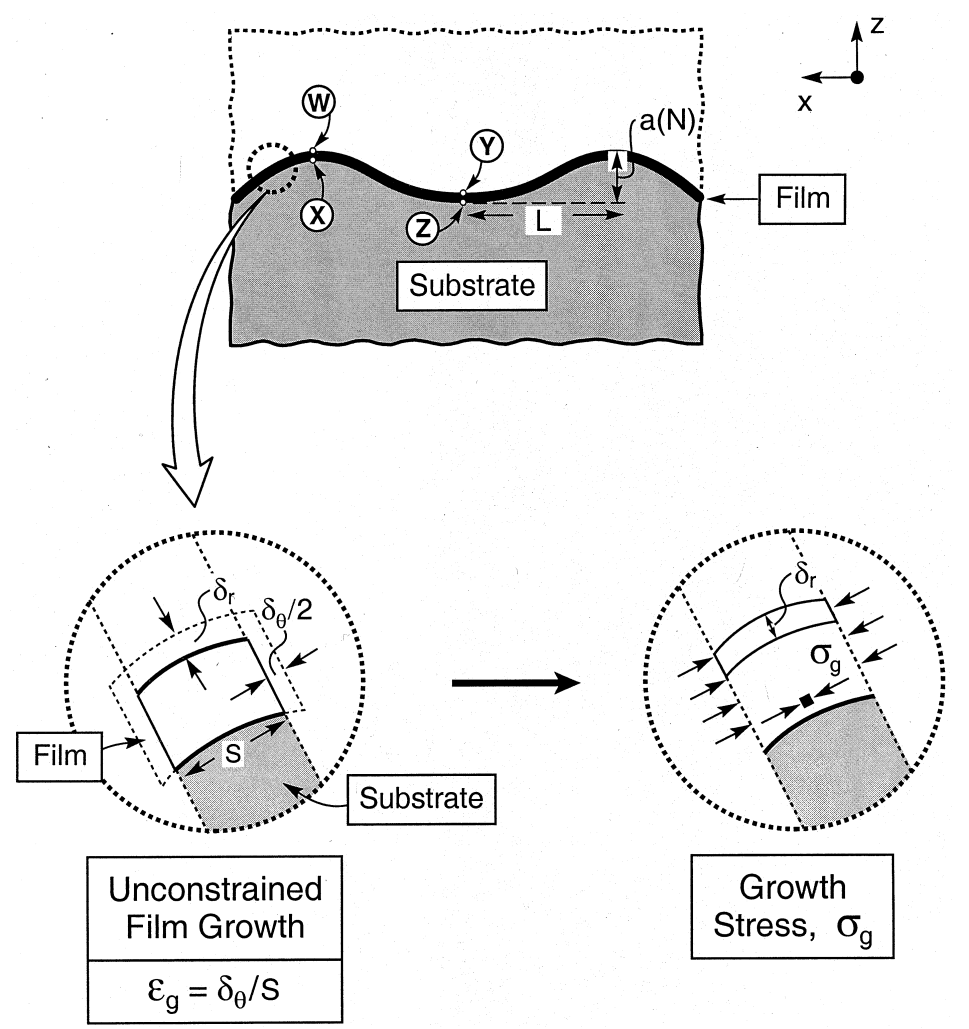

Fig. 3. The geometry subject to analysis and a schematic showing the film growth strain (unconstrained) and the consequent growth stress. 
Accordingly, the temperature increase becomes, $\Delta T+\Delta T_{\mathrm{g}}$, while the temperature decrease is still $\Delta T$. In the calculation, $\Delta T_{\mathrm{g}}$ is divided into incremental steps and the associated elastic and plastic strains computed.

\subsection{General trends}

Most of the results have been obtained for a growth strain [17], $\varepsilon_{\mathrm{g}}=10^{-4}$. A few results have been obtained for smaller and larger values in order to assess the scaling with $\varepsilon_{\mathrm{g}}$. In all cases, the wavelength, $L$, is assumed to remain constant with only changes in amplitude, $\Delta a$, allowed.

The results for various initial undulation amplitudes, $a_{0} / 2 L$, are presented using the non-dimensional quantities:

$$
\Sigma \equiv \sigma_{0} / \sigma_{\mathrm{Y}}, \quad \Pi \equiv \Delta a / 2 L \varepsilon_{\mathrm{g}}
$$

where $\Delta a$ is that part of the amplitude change attributed to $\varepsilon_{\mathrm{g}}$ and $\Delta \alpha$, with the contribution arising from uniform $\alpha_{\mathrm{s}} \Delta T$ subtracted. The normalization used for $\Pi$ does not imply that $\Delta a$ is proportional to $\varepsilon_{\mathrm{g}}$, as elaborated later. The partitioned amplitude change is a natural choice, since in performing the calculations, it has been convenient to regard $\alpha_{\mathrm{s}}$ of the substrate to be zero and to use, $\Delta \alpha=\alpha_{f}-\alpha_{s}$, as the coefficient of thermal

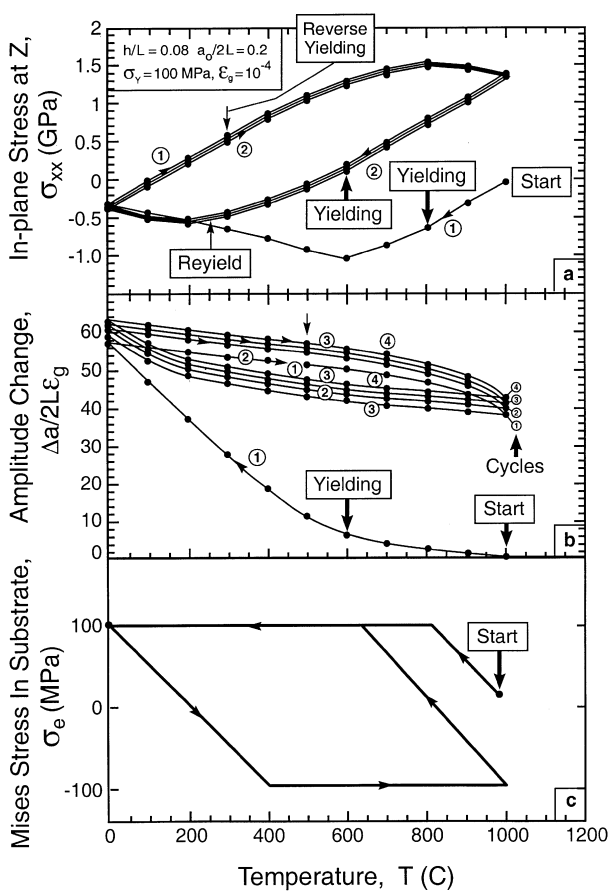

Fig. 4. Numerical calculations performed over four thermal cycles for the parameter set indicated on the inset, with $\sigma_{\mathrm{Y}}=100 \mathrm{MPa}$ : (a) in-plane stress, $\sigma_{x x}$, at site Z; (b) the change in undulation amplitude, $\Delta a$; (c) the effective, Mises, stress in the substrate (which for consistency is always taken to be positive)

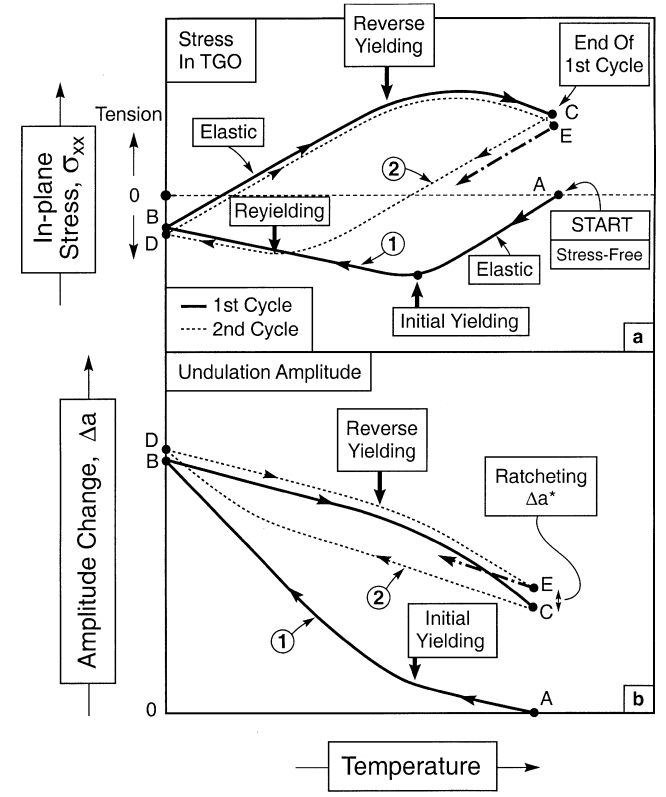

Fig. 5. A schematic of Fig. 4, highlighting the salient features.

expansion of the film. Accordingly, the total amplitude change in the first cool down, $\Delta a_{\text {total }}=$ $\Delta a+a_{0} \alpha_{\mathrm{s}} \Delta T$, can be larger than that due to $\varepsilon_{\mathrm{g}}$ and $\Delta \alpha$ : but, since it is completely reversible under temperature cycling, it makes no contribution to ratcheting. All of the calculations have been performed with the following material parameters: $E_{\mathrm{s}}=200 \mathrm{GPa}, v_{\mathrm{s}}=0.3, E_{\mathrm{f}}=400 \mathrm{GPa}, v_{\mathrm{f}}=0.2$ and $\sigma_{\mathrm{Y}}=100-500 \mathrm{MPa}$.

The initial calculations have been conducted for conditions that elicit the greatest likelihood of ratcheting. That is, results are obtained for relatively large values of $\Sigma$ and $a_{0} / 2 L$, subject to an appreciable temperature cycle, $\Delta T=1000^{\circ} \mathrm{C}$ (Figs 4 and 5). Typical results over four cycles for the in-plane stress in the film, $\sigma_{x x}$ [Fig. 4(a)], at site

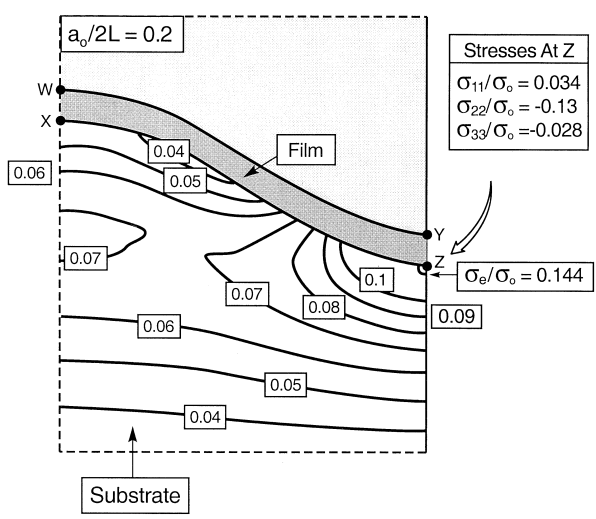

Fig. 6. Contours of equivalent stress $\sigma_{\mathrm{e}}$ induced in the substrate (normalized by the misfit stress, $\sigma_{0}$ ) for the same parameter set used to obtain Fig. 4. The stresses have been calculated at ambient after one cycle. 
$\mathrm{Z}$ (see Fig. 3), and for the change in undulation amplitude, $\Pi$ [Fig. 4(b)] illustrate the salient characteristics. The corresponding stresses in the substrate, at Z, are plotted on Fig. 4(c). The distribution of stresses in the substrate that lead to yielding are indicated on Fig. 6 for one of the cases used in the study. The magnitudes are those existing at room temperature. Note that the largest stresses are immediately beneath the valley in the film, so that yielding initiates at this site. The consequence for the stresses in the film will be addressed later.

To facilitate visualization and understanding, the key phenomena are shown in schematic form on Fig. 5. The system is stress-free at the beginning, starting at high temperature (coordinate A). Upon cooling, the initial development of $\sigma_{x x}$ and $\Delta a$ occur elastically. With further cooling, when the stresses induced in the substrate next to the film attain its yield strength [Figs 4(c) and 6], plastic deformation commences (denoted "initial yield" on Fig. 5). The associated plastic strains allow the film at the valley to displace downward. Upon yielding, the rate of stress development $\left|\mathrm{d} \sigma_{x x} / \mathrm{d} T\right|$ decreases: whereas the displacement rate, $|\mathrm{d} \Delta a / \mathrm{d} T|$ increases. For the specific parameters chosen to obtain the results on Fig. 4, even the actual stress level decreases; that is, it becomes less compressive with continued cooling. Upon re-heating (from coordinate B), the initial response is elastic. With further heating, reverse yielding initiates, as indicated on Figs 4 and 5. Thereafter, again, $\left|\mathrm{d} \sigma_{x x} / \mathrm{d} T\right|$ decreases and $|\mathrm{d} \Delta a / \mathrm{d} T|$ increases. At the end of the first cycle (coordinate $\mathrm{C}$ ) the stress is now non-zero (tensile in this case) and there has been an increase, $\Delta a^{*}$, in the undulation amplitude. Before embarking on another cycle, an increment of growth strain, $\varepsilon_{\mathrm{g}}$, is imposed on the film. This is manifest as a small increment in the undulation amplitude. On the second cycle $(\mathrm{CDE})$, the process repeats, but along a slightly different path. The consequence is a somewhat smaller stress and larger displacement at E than at $\mathrm{C}$.

\subsection{Ratcheting amplitude}

The increase in undulation amplitude during the initial few cycles is not, in itself, particularly significant. The important issue is whether the amplitude change, $\Delta a^{*}$, continues with each cycle and ratchets, or whether it damps-out after only a few cycles, resulting in shakedown. These alternative responses may be explored by repeating the thermal cycling and monitoring the change in $\Delta a^{*}$ that arises with each cycle. Examples are shown on Fig. 7(a) for two cases. One example pertains to a zero growth strain, while the other uses $\varepsilon_{\mathrm{g}}=10^{-4}$. For the former, the ratcheting rate, $\mathrm{d} \Delta a^{*} / \mathrm{d} N$, decreases with each cycle and essentially stops after 10 cycles, resulting in shakedown. Conversely, when the growth strain is included, ratcheting settles into a steady state after about 10 cycles. This result affirms the assertion made in Section 2 that a growth strain is needed to realize steady-state ratcheting. This steady-state response is the one that leads to the large overall displacements that govern cracking and failure in the top layer [Fig. 1(b)]. Repeating the calculations for a much larger substrate yield strength $(800 \mathrm{MPa})$ changes the response (Fig. 8). Now, the behavior is strictly (linear) elastic, except
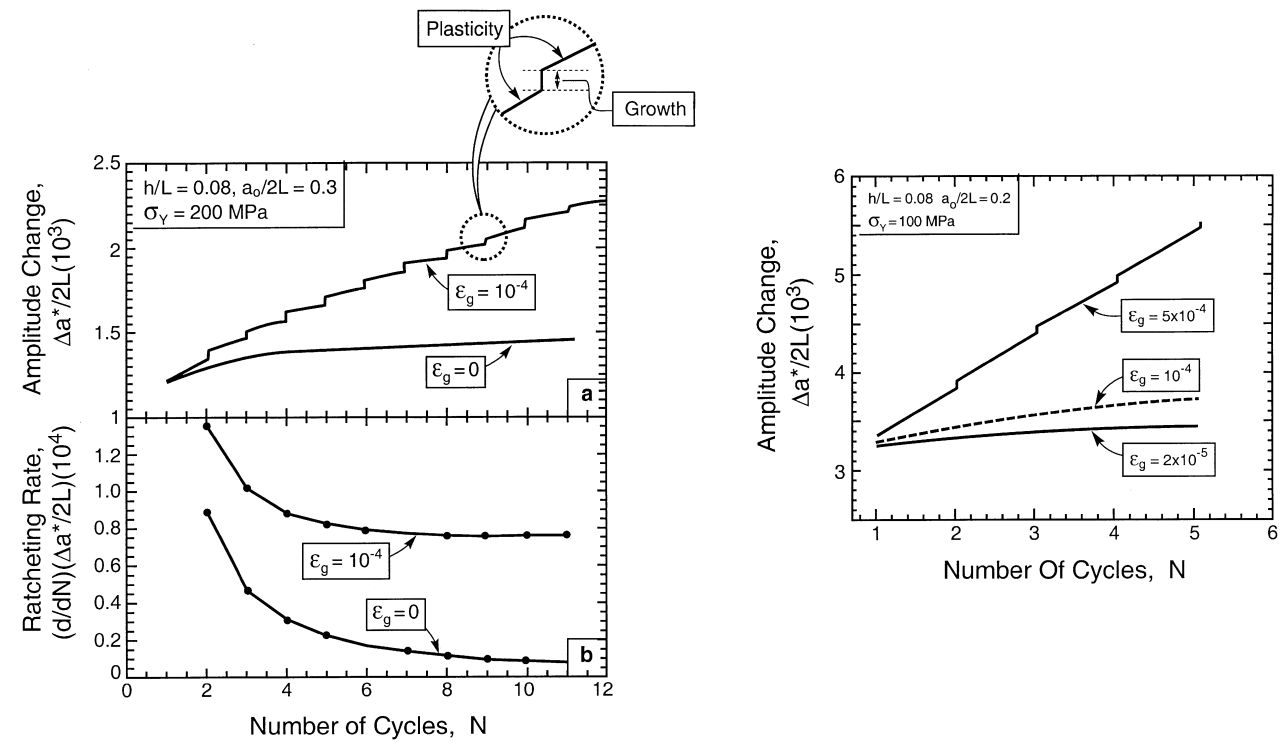

Fig. 7. (a) The effect of the growth strain, $\varepsilon_{\mathrm{g}}$, on the amplitude change and ratcheting rate at ambient temperature for the parameter set indicated on the inset. (b) The influence of growth strain on the ratcheting rate. 


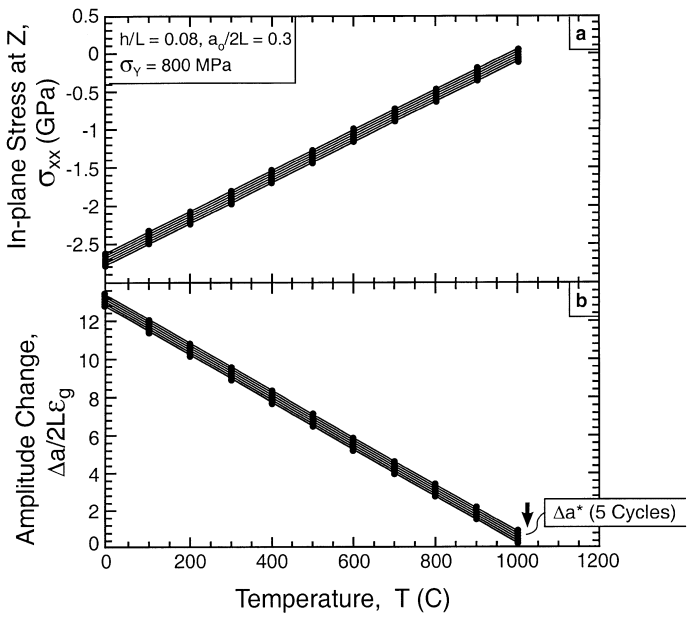

Fig. 8. Corresponding results to those in Fig. 4 for a higher yield strength substrate, $\sigma_{\mathrm{Y}}=800 \mathrm{MPa}$.

for the addition of the growth strain at the end of each cycle.

The effect of varying the growth strain is shown on Fig. 7(c). As expected, the steady-state ratcheting rate, $\mathrm{d} \Delta a^{*} / \mathrm{d} N$, increases as $\varepsilon_{\mathrm{g}}$ increases. Within the small range studied, the scaling is not quite linear. That is, an increase in $\varepsilon_{\mathrm{g}}$ by a factor of five increases the ratcheting rate by a smaller factor (three to four). A subsequent study would be needed to examine the relevant scaling.

The $\Delta a^{*}$ results obtained from many different calculations, all at a fixed growth strain $\left(\varepsilon_{\mathrm{g}}=10^{-4}\right)$, can be summarized in the manner depicted on Fig. 9. This figure expresses the amplitude changes as a function and of the stress ratio, $\Sigma \equiv \sigma_{0} / \sigma_{\mathrm{Y}}$. It distinguishes the contributions to the amplitude change per cycle from growth and cyclic plasticity. The former is invariant, while the latter increases as the stress ratio increases. For the undulations used

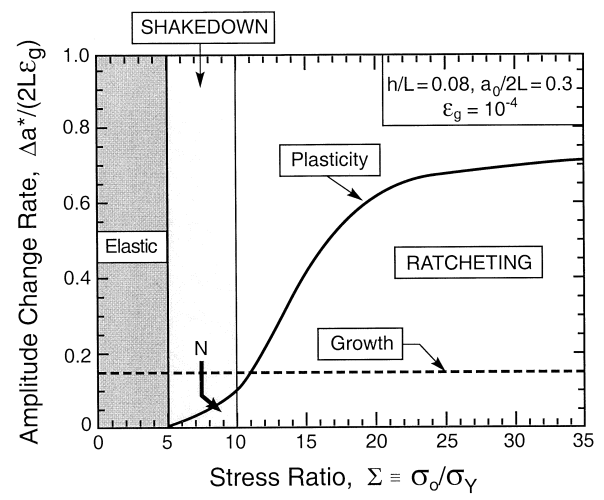

Fig. 9. A map of the three response domains using the amplitude change rate and stress ratio as coordinates. For TGO formed on superalloys, the misfit stress is almost invariant. The abscissa is thus affected largely by the yield strength of the substrate, in the sense that lower yield strength substrates are more prone to ratcheting. in these calculations, the $\Delta a_{*}$ from plasticity is zero when $\Sigma<5$ corresponding to the elastic response shown on Fig. 8. When $5<\Sigma<9$, the plasticity component reduces with each cycle and becomes zero after about 10 cycles, reflecting shakedown. At $\Sigma>9$, the plasticity component persists: indicative of the ratcheting shown in Fig. 4. These three domains are distinguished on the figure.

The effect of the initial undulation amplitude, $a_{0} / 2 L$, on the three domains, calculated using the same scheme, is presented on Fig. 10. As expected from earlier studies [3], the smaller the initial undulation, the higher the stress ratio, $\Sigma$, needed to activate ratcheting (rather than shakedown). The pertinence of these specific stress ratios is further addressed in Section 4.

\subsection{Stresses in the film}

The in-plane stresses that develop in the film as a consequence of thermal cycling are location sensitive. But after the first cycle, they remain largely invariant with subsequent cycling. These two effects are illustrated on Figs 11 and 12. The results in Fig. 11 refer to the $\sigma_{x x}$ stresses in the film at the four sites (W, X, Y, Z) shown on Fig. 3. For clarity of presentation, only the first and fifth cycles are shown. The first feature to note is that the stresses are non-uniform through the film thickness, indicative of local bending. At the undulation peak (W, $\mathrm{X}$ ), the stresses at the top of the film are tensile at the temperature maximum, but about zero at the bottom. This differential reflects a bending moment that tends to increase the undulation amplitude, consistent with the overall displacements. At the valleys $(Y, Z)$, both sides of the film are in tension at the maximum in the temperature, indicative of a stretching enabled by the plastic deformation of the substrate at this site (Fig. 7). In addition, a bending deformation accounts for the larger tensile stress at

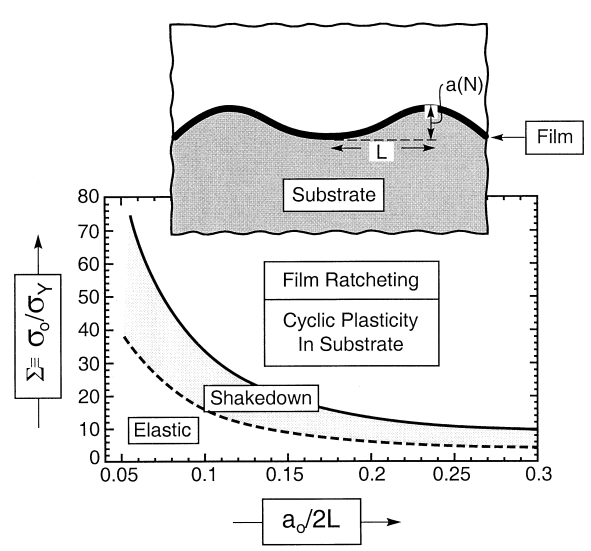

Fig. 10. The effect of initial undulation amplitude on the three domains shown on Fig. 9. The parameters used are those shown on the inset of Fig. 9. 

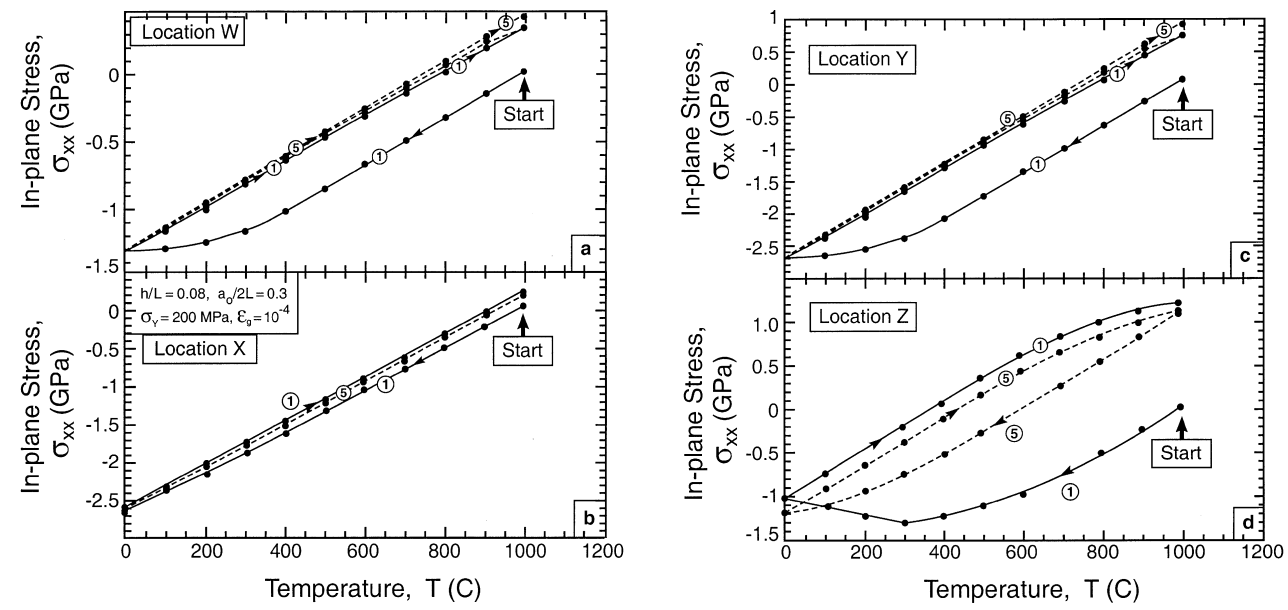

Fig. 11. In-plane stress in the first and fifth cycles at the four sites (W, X, Y, Z) shown on Fig. 3.

$\mathrm{Z}$ than at $\mathrm{Y}$; again consistent with displacements that increase the undulation amplitude. These tensile stresses may induce film cracking [9].

The relative invariance of the stress levels with the number of cycles, when ratcheting occurs, is illustrated on Fig. 12. This contrasts with the trend in the displacements, because plasticity redistributes the stresses induced by the growth strain.

\section{CONCLUDING COMMENTS}

The results summarized on Fig. 10 provide general guidelines for anticipating the combination of circumstances that lead to ratcheting; particularly the effects of geometry, $a_{0} / 2 L$, and of the stress ratio, $\sigma_{0} / \sigma_{\mathrm{Y}}$. Some corresponding effects of the growth strain, $\varepsilon_{\mathrm{g}}$, have been illustrated in Fig. 7(a). The most basic result is that steady-state ratcheting requires a growth strain in each thermal cycle. Another key finding is that the undulations must exceed a critical relative amplitude, $a_{\mathrm{c}} / L$, before they exhibit ratcheting. Moreover, this critical amplitude becomes smaller the lower the yield strength of the substrate material immediately adjacent to the film. Undulations smaller than $a_{\mathrm{c}} / L$ ex-

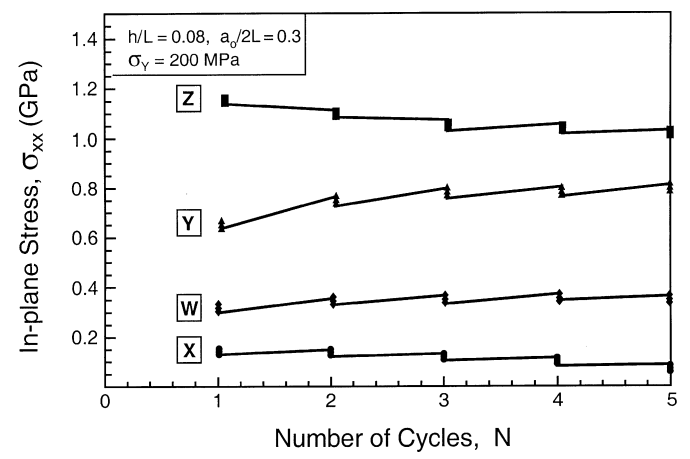

Fig. 12. The change in stress at each of the sites W, X, Y, $\mathrm{Z}$ over five cycles. perience shakedown and remain geometrically stable. The growth strain also exerts a central influence on $a_{\mathrm{c}}$. This is the least understood aspect of film growth upon oxidation [18]. New studies of this phenomenon are warranted.

For the particular case of aluminum oxide films growing on Ni-based materials, the stress ratio $\Sigma$ merits some further discussion. With $\sigma_{0}$ being about $3 \mathrm{GPa}[20]$ and the initial yield strength of the bond coat material being about $600 \mathrm{MPa}$, Fig. 10 indicates that the likelihood of ratcheting should be small, since $a_{\mathrm{c}}$ is large $(>0.6 L)$. However, as the film thickens, the $\mathrm{Al}$ concentration in the material immediately beneath the film decreases [16], because it is consumed by the growing film. This depletion causes a phase change in the alloy that may reduce its yield strength immediately beneath the oxide film. There is a corresponding reduction in $a_{\mathrm{c}}$ because of the associated change in $\Sigma$. Experimental studies that explicitly address the critical amplitude are in progress. These assessments address changes in wavelength (not just amplitude) that may occur upon ratcheting, which have not been allowed in the present calculations.

The tensile stresses predicted to develop in the film are much larger than those that would arise in practice, because creep in the film at the higher temperatures would relax them appreciably [18]. To some extent, therefore, there is competition between creep and cracking of the film during the high temperature portion of the cycle. Moreover, stress relaxation by creep would affect the growth strain and must influence the ratcheting specifics. Introducing film creep in a meaningful manner constitutes the next key step in the understanding of ratcheting.

A durability model could clearly be built from the present results for $\Delta a^{*}$ by relating this displacement to the crack opening in the TBC above the undulation [Fig. 1(b)]. The associated mechanics are addressed in another article. 


\section{REFERENCES}

1. Evans, A. G., He, M. Y. and Hutchinson, J. W., Acta mater., 1997, 45, 3543.

2. Suo, Z., J. Mech. Phys. Solids, 1995, 43, 829.

3. He, M. Y., Evans, A. G. and Hutchinson, J. W. Physica status solidi, 1998, 166, 19.

4. Tolpygo, V. K. and Clarke, D. R., Acta mater., 1998, 46, 5153.

5. Quadakkers, W. J., Holzbrecher, H., Briefs, K. G and Beske, H., Oxidation Metals, 1989, 32, 67.

6. Stott, F. H. and Wood, G. H., Mater. Sci. Engng, 1987, 87, 267.

7. Gong, X.-Y. and Clarke, D. R., Oxidation Metals, 1998, 50, 355.

8. Clarke, D. R. and Pompe, W., Acta mater., 1999, 47, 1749.

9. Tolpygo, V. K. and Clarke, D. R., Acta mater., 1999 , 47, 3589 .
10. Suo, Z., Unpublished research, Princeton University.

11. Tolpygo, V. K. and Clarke, D. R., to be published.

12. Jansson, S. and Leckie, F. A., J. Mech. Phys. Solids, 1992, 40, 593.

13. Hassan, T., Zhu, Y. and Matzen, V. C., Int. J. Pressure Vessels Piping, 1998, 75, 643.

14. Bree, J., J. Strain. Analysis, 1967, 2, 226.

15. Bree, J., J. Strain. Analysis, 1968, 3, 122.

16. Stiger, M. J., Yanar, N. M., Topping, M. G., Pettit, F. S. and Meier, G. H., Z. Metallk., 1999, 90, 1069.

17. Tolpygo, V. K., Dryden, J. and Clarke, D. R., Acto mater., 1998, 46, 927.

18. Tolpygo, V. K. and Clarke, D. R., Oxidation Metals, 1998, 49, 187.

19. Johnson, C. A., Ruud, J. A., Bruce, R. and Wortman, D., Surf. Coatings Technol., 1998, 108/109, 80.

20. Lipkin, D. M. and Clarke, D. R., Oxidation Metals, 1996, 45, 267. 